\title{
Influence of Viscosity in Fluid Atomization with Surface Acoustic Waves
}

\author{
Andreas Winkler'1, Paul Bergelt1, Lars Hillemann², Siegfried Menzel1 \\ ${ }^{1}$ IFW Dresden, SAWLab Saxony, Dresden, Germany \\ ${ }^{2}$ TU Dresden, Institute for Process Engineering and Environmental Technology, Dresden, Germany \\ Email: a.winkler@ifw-dresden.de
}

How to cite this paper: Winkler, A., Bergelt, P., Hillemann, L. and Menzel, S. (2016) Influence of Viscosity in Fluid Atomization with Surface Acoustic Waves. Open Journal of Acoustics, 6, 23-33.

http://dx.doi.org/10.4236/oja.2016.63003

Received: August 26, 2016

Accepted: September 27, 2016

Published: September 30, 2016

Copyright $\odot 2016$ by authors and Scientific Research Publishing Inc. This work is licensed under the Creative Commons Attribution International License (CC BY 4.0).

http://creativecommons.org/licenses/by/4.0/

\begin{abstract}
In this work, aqueous glycerol solutions are atomized to investigate the influence of the viscosity on the droplet size and the general atomization behavior in a setup using standing surface acoustic waves (sSAW) and a fluid supply at the boundary of the acoustic path. Depending on the fluid viscosity, the produced aerosols have a monomodal or polymodal size distribution. The mean droplet size in the dominant droplet fraction, however, decreases with increasing viscosity. Our results also indicate that the local wavefield conditions are crucial for the atomization process.
\end{abstract}

\section{Keywords}

Surface Acoustic Waves, Atomization, Aerosol, High Viscosity,

Droplet Size Distribution, Wavefield

\section{Introduction}

Surface Acoustic Wave (SAW) fluid atomizers have the demonstrated ability to generate droplets without the need for moving parts or nozzles. Their great potential for integration is expected to lead to an economic production of hand-held and even disposable devices, with a single functionality or integrated in more complex superior systems. The principle of fluid atomization using surface acoustic waves on piezoelectric chips was demonstrated by Kurosawa et al. in 1995 [1] [2]. Since then, this phenomenon has been investigated regarding the underlying physical phenomena [3]-[6] or possible applications, including inhalation therapy [7] [8], olfactory displays [9], micro- and nanoparticle synthesis [10]-[12], thin film deposition [13]-[15] and mass spectroscopy of non-volatile fluids [16]-[18].

In principle, SAW-based fluid atomization is the result of the interaction of an acoustic wave on the surface of a piezoelectric substrate with a fluid placed in its prop- 
agation path. The droplet generation appears to be a near field effect, taking place in a film of liquid with a characteristic thickness in the range of the acoustic wavelength, generated and stabilized by the SAW out of a parent liquid body. The most important effect associated with such a liquid film is its acoustically-driven transient development, spreading and thinning (termed "acoustowetting") [19] [20]. The geometrical properties of the fluid film are expected to depend on the wavefield, the method and position of fluid supply and the fluid properties. However, the dimensions of the fluid film with a thickness in the order of magnitude of the acoustic wavelength and its dynamic behavior are difficult to analyze and more thorough investigations are necessary in order to optimize the acoustofluidic interaction for future devices.

Existing studies on the atomization mechanism concentrated mainly on low viscosity fluids including water and basic organic solvents. However, fluids including mono-/ polymer solutions and those used in olfactory systems or in sol-gel deposition can exhibit a significantly higher viscosity. The fluid viscosity hereby is expected to have a significant influence on the atomization mechanism and the obtainable droplet size. This work concentrates on the atomization of aqueous glycerol solutions as model fluids with high viscosity.

\section{Experimental}

Pairs of interdigital transducers ( $\lambda / 4$ IDT, $\lambda=20$ to $120 \mu \mathrm{m}, 28$ to 50 electrode pairs, 0.5 $\mathrm{mm}$ aperture) matched to $50 \Omega$ impedance by design and made of subsequent layers of $\mathrm{Ti}(5 \mathrm{~nm})$ and highly-textured $\mathrm{Al}(295 \mathrm{~nm})$ were prepared via electron-beam evaporation and lift-off technique on a $128^{\circ} \mathrm{YX}-\mathrm{LiNbO}_{3}$ substrate $\left(128^{\circ}\right.$ rotated Y-cut Lithium Niobate with X-propagation direction, $8 \mathrm{~mm} \times 9 \mathrm{~mm}$ or $8 \mathrm{~mm} \times 14 \mathrm{~mm}$ chip size). In the used layout, the IDTs oppose each other with a distance of $6 \mathrm{~mm}$ (delay line) for standing SAW (sSAW) excitation. A $1000 \mathrm{~nm}$ thick $\mathrm{SiO}_{2}$ layer [21] was sputter-deposited on the chip surface in order to inhibit aluminum corrosion and to establish a chemically compatible surface on the piezoelectric substrate. The chip was placed on a CNC milled and anodized aluminum platform using an interlayer of thermally conductive foil (TFO-X200-SI, HalaContec GmbH \& Co. KG). The chips were electrically contacted and mechanically retained using two printed circuit board conductor plates with strip lines matched to $50 \Omega$ impedance and gold-coated spring pins. Radio frequency (rf) signals were supplied at the working frequency of the transducers, i.e. at minimum power reflection, via SMA cables from a dual-channel Power SAW F20 signal source (BelektroniG $\mathrm{GmbH}$, Germany) with a load power of up to approximately 3.5 W supplied to each IDT. A sketch of the chip setup is shown in Figure 1(a).

Subsequent to the positioning of a stainless steel capillary $\left(45^{\circ}\right.$ angled dosage tip, Gauge 30, Nordson Co.) at the boundary of the acoustic wavefield and the excitation of sSAW, the fluid was delivered to the chip surface. Aqueous glycerol solutions (0 to $85 \% \mathrm{v}$ glycerol content) were used as model fluids in this study. The fluid flow rate was maintained constant up to $400 \mu \mathrm{l} / \mathrm{min}$ via a neMESYS syringe pump (Cetoni $\mathrm{GmbH}$, Germany). Characterization of the aerosol with respect to its droplet size distribution in 


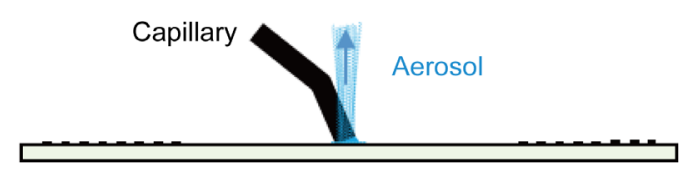

Side view

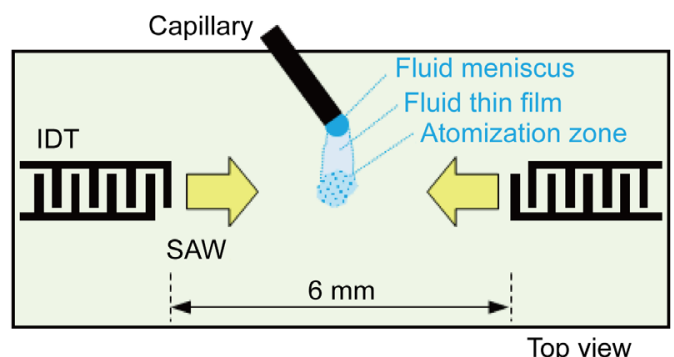

(a)

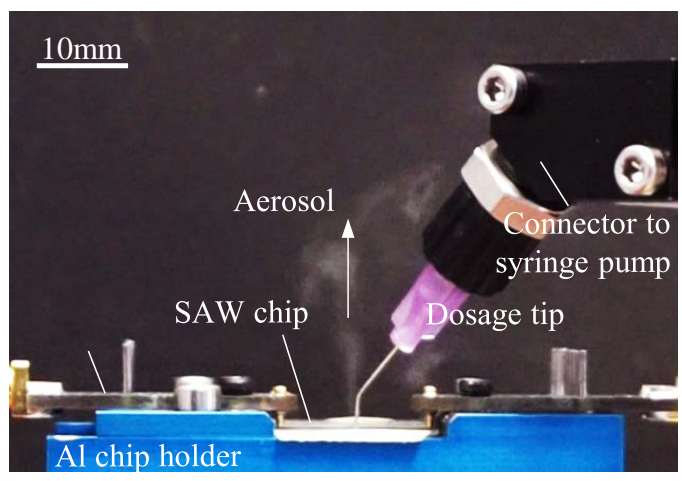

(b)

Figure 1. sSAW atomization setup. (a) Sketch; (b) Side view on atomization of a highly viscous aqueous glycerol solution ( $85 \%$ glycerol).

the range of 1 to $100 \mu \mathrm{m}$ was carried out using a Helos KR laser diffractometer (Sympatec $\mathrm{GmbH}$, Germany). Here, the droplet size distribution of the aerosol jet was measured for $90 \mathrm{~s}$ in a distance of $30 \mathrm{~mm}$ to the chip surface with a laser spot of approximately $20 \mathrm{~mm}$ diameter. An extraction unit was used to collect the aerosol above the measurement spot in order to prohibit fluid condensation on the diffractometer lenses. Droplet sizes were calculated using the Mie scattering theory [22]. The amplitude distributions of selected IDTs were measured with a UHF 120 laser Doppler vibrometer (Polytec GmbH, Germany). The measurement point density used here was 2.1 points/wavelength in $\mathrm{y}$-direction and $500 \mu \mathrm{m}$ in $\mathrm{x}$-direction. Fluid contact angles were measured using a CAM 101 setup (KSV Instruments Inc.).

\section{Results \& Discussion}

\subsection{Wavefield and Diffraction}

In order to investigate the influence of the viscosity on the aerosol generation process, different aqueous glycerol solutions were used as model fluids. The non-toxic and over a large frequency-range Newtonian fluids [23] are ideally suited for this task. By changing the water-glycerol ratio between 0 to $85 \%$ v, the viscosity can be changed from 
$\eta=1$ to approx. $124 \mathrm{mPas}$. Thereby, only slight changes are measured in the density ( $\rho$ $=1$ to $\left.1.22 \mathrm{~g} / \mathrm{cm}^{3}\right)$ and the surface tension $(\gamma=72.1$ to $64.4 \mathrm{mN} / \mathrm{m})$ [24]. The influence of the conductivity of the fluids on the electrical field of the SAW can be neglected in a first approximation due to the high dielectricity $\left(\varepsilon_{r} \approx 20-40\right)$ of glycerol at $\mathrm{MHz}$ frequencies and moderate temperatures [25].

In previous work, the advantages of the boundary fluid supply approach in SAWbased fluid atomization [26] were already demonstrated, including (I) the prevention of a larger fluid volume in the SAW propagation path, (II) a spatial separation of atomization zone and fluid supply and (III) minimized interaction between SAW and the means of fluid supply. Therefore, the experiments described here were carried out using sSAW with a fluid supply via a stainless steel capillary positioned at the boundary of the acoustic path (Figure 1). Figure 2 shows a tilted camera view on the chip surface during such an experimental setup during the atomization of water (see also supplementary video 1). The locations of the capillary fluid supply, the meniscus, the fluid film and the atomization zone are clearly visible. Moreover, the oscillation of the meniscus due to Eckart streaming, the high temporal stability of the aerosol beam and the spatial stability of the atomization zone can be observed.

It is assumed, that the SAW wavefield, i.e. the lateral distribution of amplitude (and phase), is the key to these issues. In Figure 3, the amplitude distributions of individual IDTs with wavelengths of 30 to $90 \mu \mathrm{m}$ and a constant aperture of $0.5 \mathrm{~mm}$ are shown. As the IDTs used in this study have a separation of $6 \mathrm{~mm}$, they are located to the left and to the right of the diagrams or at position 0 and $6 \mathrm{~mm}$, respectively (indicated in Figure 3 (b) by red bars). The fluid was supplied in the chip middle ( $\mathrm{x}$ - or propagation-direction), at the boundary of the acoustic path (y-direction, blue region). While Figure 3(a) shows the amplitude distribution of a travelling wave, excited by the left IDT, the standing wave amplitude could also be obtained. In order to get the approximate maximum amplitude distribution $\mathrm{u}_{3, \max }$ for the used standing wave setup, the travelling wavefields can be horizontally mirrored and combined by vectorial addition (as in Figure 3(b)).
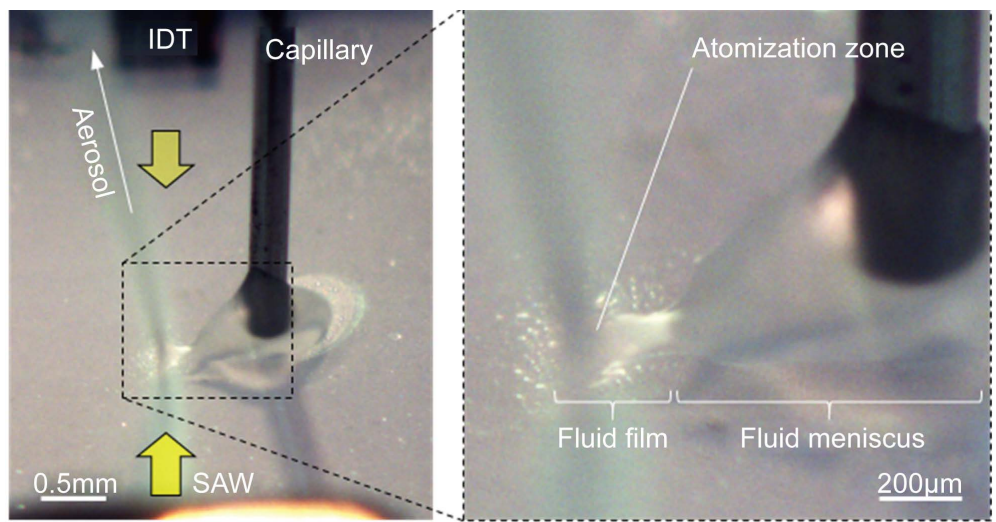

Figure 2. Tilted view on chip surface during atomization: overview and inset showing the atomization zone with fluid meniscus, fluid film and aerosol stream. 
(a) SAW

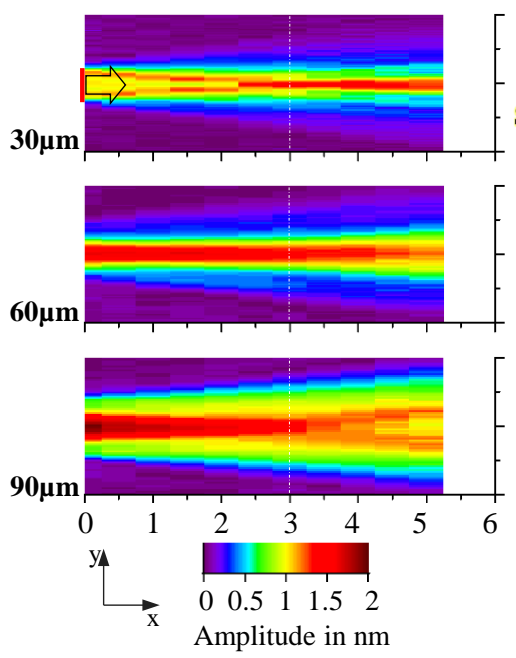

(b) SSAW

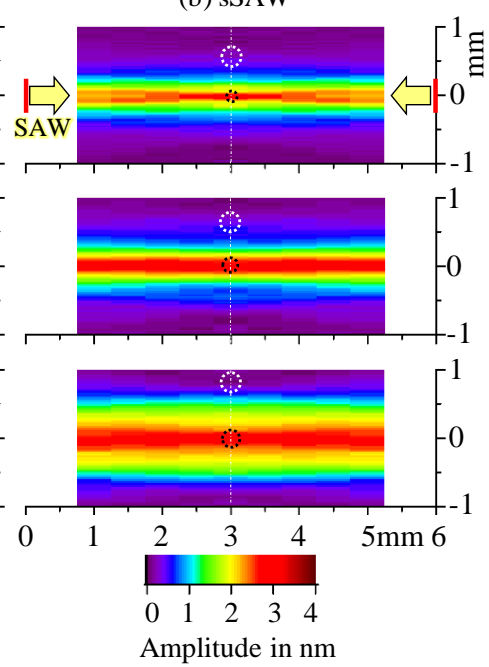

Figure 3. SAW amplitude distributions for IDTs with three different wavelengths and an aperture of $0.5 \mathrm{~mm}(2 \times 100 \mathrm{~mW}$ load power, damping mass on chip edges): (a) Measured amplitude $u_{3, \max }$ for travelling SAW (one side of a bidirectional IDT positioned at $\mathrm{x}=0 \mathrm{~mm}$ ); (b) Standing SAW (sSAW) amplitude $\mathrm{u}_{3, \max }$ (between two IDTs) obtained by vectorial addition of two mirrored distributions from (a); approximate positions of fluid supply (white dotted circle), atomization zone (black dotted circle) and IDT position (red bar) indicated.

The wavefield properties, e.g. the existence and distribution of amplitude minima/ maxima or their lateral extension, are mainly determined by SAW diffraction. It was found that the lateral extension of the propagation path in $y$-direction as well as the existing amplitude and amplitude gradient distribution depend strongly on the SAW wavelength and the number of finger electrodes-for fixed aperture, electrode height and electrode material. Thereby, the amplitude and its gradient is responsible for the transport of the fluid into the atomization zone. The distance between the position of the fluid supply at the boundary of the acoustic path (marked by white circle) and the atomization zone in the center (black circle) here varies between approximately 0.3 and 0.7 $\mathrm{mm}$, in $3 \mathrm{~mm}$ distance from the IDTs, respectively. This leads to differing fluid film dimensions for the different setups or-more general-for different diffraction conditions, which could be very relevant for practical applications. The amplitude in the beam center and the lateral extension of the amplitude maximum in the atomization zone also depend on the diffraction conditions. Both effects could generate changed geometrical fluid film conditions in the atomization zone and, hence, changed atomization conditions, including the maximum flow rate, the droplet size distribution and the droplet impulse.

\subsection{General Observations}

The fluids viscosity itself influences the atomization process in various ways. At a fixed load power and setup, the increase of the viscosity seems to limit the applicable SAW wavelength. While water could be atomized in a broad wavelength range (20 to $120 \mu \mathrm{m}$ ), 
a solution with $30 \% \mathrm{v}$ glycerol content could only be atomized using wavelengths of 30 to $90 \mu \mathrm{m}$. A further increase of glycerol concentration limited the usable wavelength range to 30 to $60 \mu \mathrm{m}$, or frequencies of approximately 65 to $135 \mathrm{MHz}$, respectively. Again, the wavefield or more specific the maximum surface-normal amplitude $\mathrm{u}_{3, \max }$ and its lateral distribution may be responsible for this. An increase in viscosity was also found to reduce the maximum possible fluid flow rate as well as the height of the aerosol jet. While water can be atomized in the setup with flow rates up to $400 \mu \mathrm{l} / \mathrm{min}$ and approx. $50 \mathrm{~mm}$ height, a maximum flow rate of $30 \mu \mathrm{l} / \mathrm{min}$ at $20 \mathrm{~mm}$ aerosol height was possible for the highest viscosity glycerol solution. Here, the increased viscous damping of the longitudinal pressure wave induced in the fluid film may be causative, as it may reduce the amplitude of capillary waves at the fluid-gas interphase and thus, it may hinder the droplet breakup and reduce their initial speed.

It should also be mentioned, that an increased viscosity could furthermore lead to more localized dissipation of the acoustic energy associated with significant heat production in the fluid film and local temperature increase in the presence of insufficient heat conduction to the chip holder. In a recent study, temperatures of up to $90^{\circ} \mathrm{C}$ in the atomization zone were measured even during the atomization of low viscous fluids [27]. Such high temperatures could lead to damage of the chip or of fluidic components. However, this study was carried out without any heat dissipation means and the temperature of the almost transparent setup was measured with by an unreliable optical technique. Another study came to the opposite result, namely that delicate proteins and even yeast cells could be atomized without damage [28]. While further investigations of the local temperature in the atomization zone are necessary, an effective heat dissipation through the SAW chip has to be guaranteed in order to minimize the influence of the temperature on the atomization process.

\subsection{Droplet Size Distribution}

Figure 4(a) shows the measured droplet size distributions for six different glycerol solutions at fixed wavelength, flow rate and load power. All distributions are modal, i.e. only certain droplet sizes exist, causing peaks of different height and width. For high glycerol content $(\geq 50 \% \mathrm{v})$, a monomodal distribution was observed, whereby the size of the produced droplets and its standard deviation are gradually decreasing with increasing viscosity. At low glycerol content $(0$ and $30 \% \mathrm{v})$, several droplet fractions exist, indicating different physical droplet production mechanisms. For pure water, three additional droplet fractions are visible. The origin of the small droplets $(<2 \mu \mathrm{m})$, also observed for water in earlier studies [3] [15], could not be clarified so far. A droplet breakup in the gas phase could be neglected, as the droplet's mean Weber number (We < 0.1 ) is too low, even for the basic vibrational droplet breakup [29]. The main peak itself shows a shoulder towards lower droplet sizes (also existent for the $30 \% \mathrm{v}$ solution), which origin is also unclear.

Larger droplets $(>30 \mu \mathrm{m})$ are with high probability the product of fluid jetting, occurring when the fluid volume present in the acoustic propagation path exceeds the 


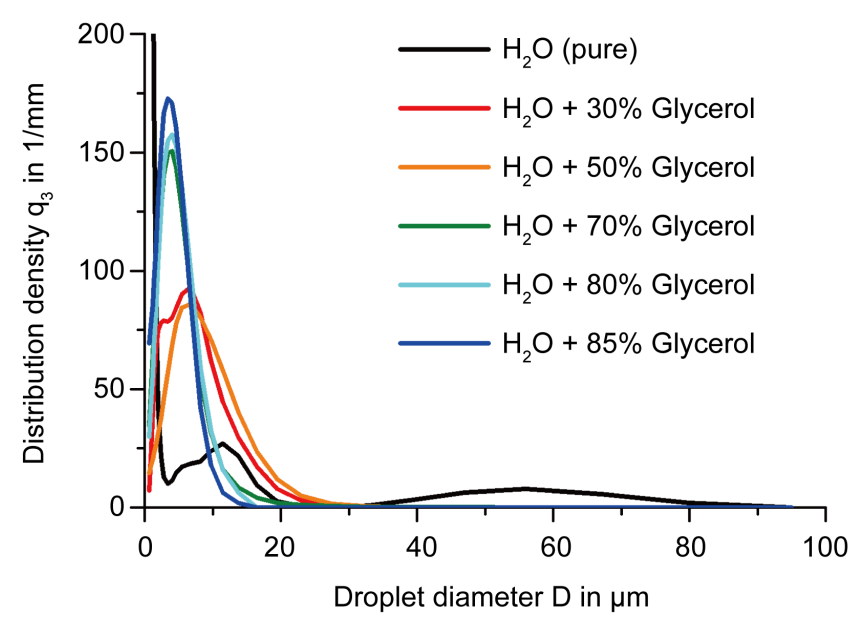

(a)

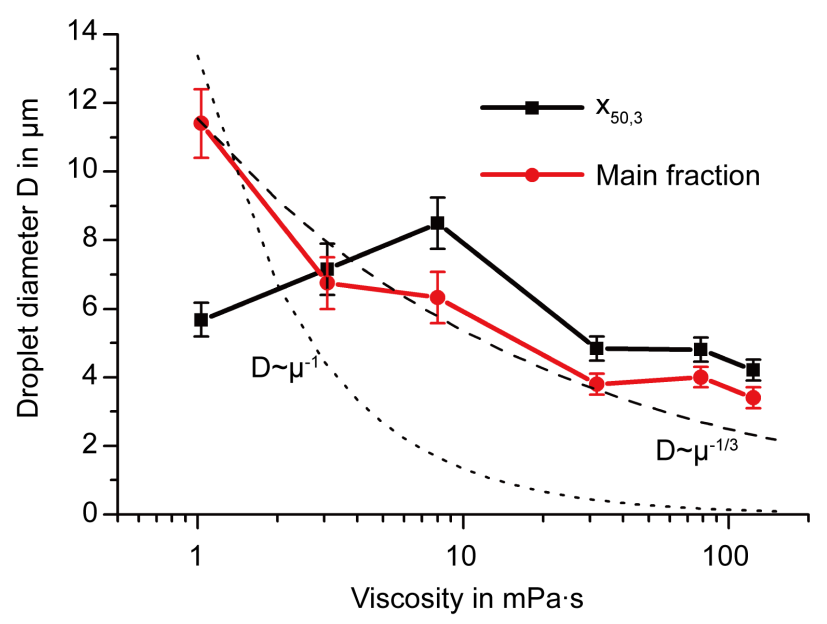

(b)

Figure 4. Droplet size distribution for different aqueous glycerol solutions atomized using sSAW $(\lambda=60 \mu \mathrm{m}, 30 \mu \mathrm{l} / \mathrm{min})$. (a) Size distribution density; (b) Peak value of the main droplet fraction and median value $\left(\mathrm{x}_{50,3}\right)$, fitted trends for thedroplet size proportionality to viscosity indicated by dotted $(D \sim 1 / \mu)$ or dashed $\left(D \sim 1 / \mu^{1 / 3}\right)$ lines.

film condition, i.e. for fluid dimensions larger than the wavelength of the longitudinal wave. Jetting was observed to occur at sufficient SAW amplitude on hydrophobic surfaces [30], i.e. for contact angles above the complementary longitudinal wave radiation angle $\left(90^{\circ}\right.$ minus the Rayleigh angle $\left.\theta_{R}\right)$, i.e. approx. $70^{\circ}$ measured from the surface. Our measurements show a linear decrease of the static contact angle on the sputterdeposited $\mathrm{SiO}_{2}$ surface with increasing glycerol concentration. While pure water shows a contact angle of approx. $55 \pm 2^{\circ}$, the contact angle reduces to approx. $42 \pm 2^{\circ}$ for an $85 \% \mathrm{v}$ glycerol solution. Larger droplets were only measured for water, which had the highest contact angle. Therefore, the pressure exerted by the longitudinal wave on the fluid-gas interphase seems to be causative: For contact angles well below $90^{\circ}-\theta_{R}$, the reflection of the travelling longitudinal pressure wave at the fluid-air interface and, thus, 
the development of a vertical longitudinal standing wave is eased. Thereby, the formation of the fluid film should be favorable. However, as the pressure exerted on the fluidgas interphase could increase the contact angle under SAW influence, a condition for jetting could be fulfilled for contact angles below, but close to $90^{\circ}-\theta_{R}$. This could be the case for the solutions with very low glycerol content. However, due to the proportionality of the droplet volume with $d^{\beta}$, only few large droplets-compared to the other fractions-are sufficient to form a measurable peak. In addition, first investigations show that the reduction of the contact angle, e.g. by appropriate functionalization of the chip surface, hinders large droplet formation. Other less probable, but possible mechanisms for large peak production could be the agglomeration of droplets in the gas phase or a change of the local refractivity due to water evaporation.

When only the droplets of the main fraction, originating from the fluid film atomization mechanism, are considered (Figure 4(b), main fraction values), a continuous reduction of the droplet size and its standard deviation with increasing viscosity can be observed. The currently existing atomization model by Collins et al. [3]

$$
D \sim \frac{\gamma \cdot H^{2}}{\mu \cdot L^{2}} \cdot \frac{W e^{\frac{2}{3}}}{f}
$$

assumes an indirect proportionality of the droplet size $D$ to the dynamic viscosity $\mu$. However, so far the model was evaluated only for water and not for fluids with higher viscosity. In addition, only travelling waves were investigated in the underlying study and the $L / H$ ratio, i.e. the ratio of fluid film length to height, is not directly applicable to the observations in a standing wave setup. Our results show an approximate proportionality of $D \sim 1 / \mu^{1 / 3}$ for the droplets of the main peak. As expected, the integration of all droplet fractions, as it is done in the median value $\mathrm{x}_{50,3}$, does not show this clear relationship.

\section{Conclusion}

In this work, the successful SAW-based atomization of highly viscous aqueous glycerol solutions was demonstrated in a setup using standing surface acoustic waves (sSAW) and a fluid supply at the boundary of the acoustic path. Thereby, the droplet size in the main droplet fraction and its standard deviation decrease with increasing viscosity. Additional peaks originating from other physical droplet production mechanisms occur for the low viscosity fluids. The aim for any application-relevant problem should therefore be the prevention of secondary droplet production mechanisms by optimization of the fluid supply, the acoustic wavefield and the surface chemistry. The applicable wavelength, the maximum fluid flow rate and the aerosol height seem to be limited by the viscosity of the fluid and the wavefield properties. Our results indicate that the local wavefield conditions are crucial for the atomization process and have to be taken into account together with the surface chemistry for design and optimization of future application-relevant devices. 


\section{Acknowledgements}

This work was supported by the by the German Research Foundation (DFG Grant WI 4140/2-1).

\section{References}

[1] Kurosawa, M., Watanabe, T., Futami, A. and Higuchi, T. (1995) Surface Acoustic Wave Atomizer. Sensors and Actuators A: Physical, 50, 69-74. http://dx.doi.org/10.1016/0924-4247(96)80086-0

[2] Kurosawa, M., Watanabe, T. and Higuchi, T. (1995) Surface Acoustic Wave Atomizer with Pumping Effect. IEEE Proceedings of Micro Electro Mechanical Systems, Amsterdam, 29 January-2 February 1995, 25-30. http://dx.doi.org/10.1109/memsys.1995.472559

[3] Collins, D.J., Manor, O., Winkler, A., Schmidt, H., Friend, J.R. and Yeo, L.Y. (2012) Atomization Off Thin Water Films Generated by High-Frequency Substrate Wave Vibrations. Physical Review E, 86, 1-9. http://dx.doi.org/10.1103/PhysRevE.86.056312

[4] Qi, A., Yeo, L.Y. and Friend, J.R. (2008) Interfacial Destabilization and Atomization Driven by Surface Acoustic Waves. Physics of Fluids, 20, 1-14. http://dx.doi.org/10.1103/PhysRevE.86.056312

[5] Taller, D., Go, D.B. and Chang, H.C. (2013) Modulated Exponential Films Generated by Surface Acoustic Waves and Their Role in Liquid Wicking and Aerosolization at a Pinned Drop. Physical Review E, 87, Article ID: 053004. http://dx.doi.org/10.1103/physreve.87.053004

[6] Ang, K.M., Yeo, L.Y., Hung, Y.M. and Tan, M.K. (2016) Graphene-Mediated Microfluidic Transport and Nebulization via High Frequency Rayleigh Wave Substrate Excitation. Lab on a Chip, 16, 3503-3514. http://dx.doi.org/10.1039/C6LC00780E

[7] Qi, A.S., Friend, J.R., Yeo, L.Y., Morton, D.A.V., McIntosh, M.P. and Spiccia, L. (2009) Miniature Inhalation Therapy Platform Using Surface Acoustic Wave Microfluidic Atomization. Lab on a Chip, 9, 2184-2193. http://dx.doi.org/10.1039/b903575c

[8] Rajapaksa, A., Qi, A.S., Yeo, L.Y., Coppel, R. and Friend, J.R. (2014) Enabling Practical Surface Acoustic Wave Nebulizer Drug Delivery via Amplitude Modulation. Lab on a Chip, 14, 1858-1865. http://dx.doi.org/10.1039/C4LC00232F

[9] Nakamoto, T., Hashimoto, K., Aizawa, T. and Ariyakul, Y. (2014) Multi-Component Olfactory Display with a Saw Atomizer and Micropumps Controlled by a Tablet Pc. Proceedings of the IEEE International Frequency Control Symposium (FCS), Taipei, 19-22 May 2014, 1-4.

[10] Alvarez, M., Friend, J.R. and Yeo, L.Y. (2008) Rapid Generation of Protein Aerosols and Nanoparticles via Surface Acoustic Wave Atomization. Nanotechnology, 19, 455103. http://dx.doi.org/10.1088/0957-4484/19/45/455103

[11] Kim, J.W., Yamagata, Y., Takasaki, M., Lee, B.H., Ohmori, H. and Higuchi, T. (2005) A Device for Fabricating Protein Chips by Using a Surface Acoustic Wave Atomizer and Electrostatic Deposition. Sensors and Actuators B-Chemical, 107, 535-545. http://dx.doi.org/10.1016/j.snb.2004.11.012

[12] Qi, A.S., Chan, P., Ho, J., Rajapaksa, A., Friend, J. and Yeo, L.Y. (2011) Template-Free Synthesis and Encapsulation Technique for Layer-by-Layer Polymer Nanocarrier Fabrication. ACS Nano, 5, 9583-9591. http://dx.doi.org/10.1021/nn202833n

[13] Murochi, N., Sugimoto, M., Matsui, Y. and Kondoh, J. (2007) Deposition of Thin Film Using a Surface Acoustic Wave Device. Japanese Journal of Applied Physics Part 1-Regular 
Papers Brief Communications \& Review Papers, 46, 4754-4759.

http://dx.doi.org/10.1143/JAP.46.4754

[14] Darmawan, M., Jeon, K., Ju, J.M., Yamagata, Y. and Byun, D. (2014) Deposition of Poly(3,4ethylenedioxythiophene)-poly(styrenesulfonate) (Pedot-Pss) Particles Using Standing Surface Acoustic Waves and Electrostatic Deposition Method for the Rapid Fabrication of Transparent Conductive Film. Sensors and Actuators A: Physical, 205, 177-185.

http://dx.doi.org/10.1016/j.sna.2013.11.016

[15] Winkler, A., Kirchner, A., Bergelt, P., Hühne, R. and Menzel, S. (2016) Thin Film Deposition Based on Microacoustic Sol Atomization (Masa). Journal of Sol-Gel Science and Technology, 78, 26-33. http://dx.doi.org/10.1007/s10971-015-3927-6

[16] Ho, J., Tan, M.K., Go, D.B., Yeo, L.Y., Friend, J.R. and Chang, H.C. (2011) Paper-Based Microfluidic Surface Acoustic Wave Sample Delivery and Ionization Source for Rapid and Sensitive Ambient Mass Spectrometry. Analytical Chemistry, 83, 3260-3266. http://dx.doi.org/10.1021/ac200380q

[17] Huang, Y., Yoon, S.H., Heron, S.R., Masselon, C.D., Edgar, J.S., Turecek, F., et al. (2012) Surface Acoustic Wave Nebulization Produces Ions with Lower Internal Energy than Electrospray Ionization. Journal of the American Society for Mass Spectrometry, 23, 1062-1070. http://dx.doi.org/10.1007/s13361-012-0352-8

[18] Monkkonen, L., Edgar, J.S., Winters, D., Heron, S.R., Mackay, C.L., Masselon, C.D., et al. (2016) Screen-Printed Digital Microfluidics Combined with Surface Acoustic Wave Nebulization for Hydrogen-Deuterium Exchange Measurements. Journal of Chromatography A, 1439, 161-166. http://dx.doi.org/10.1016/j.chroma.2015.12.048

[19] Manor, O., Rezk, A.R., Friend, J.R. and Yeo, L.Y. (2015) Dynamics of Liquid Films Exposed to High-Frequency Surface Vibration. Physical Review E, 91, Article ID: 053015. http://dx.doi.org/10.1103/physreve.91.053015

[20] Altshuler, G. and Manor, O. (2015) Spreading Dynamics of a Partially Wetting Water Film Atop a Mhz Substrate Vibration. Physics of Fluids, 27, Article ID: 102103. http://dx.doi.org/10.1063/1.4932086

[21] Winkler, A., Menzel, S.B. and Schmidt, H. (2009) Saw-Grade $\mathrm{SiO}_{2}$ for Advanced Microfluidic Devices. Proceedings of the SPIE, 7362, Article ID: 73621Q.

[22] Mie, G. (1908) Beiträge Zur Optik Trüber Medien, Speziell Kolloidaler Metallösungen. Annalen der Physik, 330, 377-445. http://dx.doi.org/10.1002/andp.19083300302

[23] Brünig, R. (2011) Modellierung Von Akustischen Dickenscherschwingern Im Frequenzbereich. PhD Thesis, Faculty of Electrical and Computer Engineering, TU Dresden, Dresden.

[24] Segur, J.B. and Oberstar, H.E. (1951) Viscosity of Glycerol and Its Aqueous Solutions. Industrial \& Engineering Chemistry, 43, 2117-2120. http://dx.doi.org/10.1021/ie50501a040

[25] Schulz, A.K. (1954) Über Die Aus Ultraschallversuchen Und Dielektrischen Messungen Ermittelte Relaxationszeit Und Ihre Abhängigkeit Von Der Viskosität. Zeitschrift für Naturforschung $A$, 9, 944-950. http://dx.doi.org/10.1515/zna-1954-1106

[26] Winkler, A., Harazim, S.M., Menzel, S.B. and Schmidt, H. (2015) Saw-Based Fluid Atomization Using Mass-Producible Chip Devices. Lab on a Chip, 15, 3793-3799. http://dx.doi.org/10.1039/C5LC00756A

[27] Hashimoto, K. and Nakamoto, T. (2015) Stabilization of Saw Atomizer for a Wearable Olfactory Display. IEEE International Ultrasonics Symposium, 1, 121-124.

[28] Qi, A., Yeo, L., Friend, J. and Ho, J. (2010) The Extraction of Liquid, Protein Molecules and Yeast Cells from Paper through Surface Acoustic Wave Atomization. Lab on a Chip, 10, 
470-476. http://dx.doi.org/10.1039/B915833B

[29] Pilch, M. and Erdman, C. (1987) Use of Breakup Time Data and Velocity History Data to Predict the Maximum Size of Stable Fragments for Acceleration-Induced Breakup of a Liquid Drop. International Journal of Multiphase Flow, 13, 741-757.

http://dx.doi.org/10.1016/0301-9322(87)90063-2

[30] Tan, M.K., Friend, J.R. and Yeo, L.Y. (2009) Interfacial Jetting Phenomena Induced by Focused Surface Vibrations. Physical Review Letters, 103, Article ID: 024501.

http://dx.doi.org/10.1103/PhysRevLett.103.024501

Submit or recommend next manuscript to SCIRP and we will provide best service for you:

Accepting pre-submission inquiries through Email, Facebook, LinkedIn, Twitter, etc. A wide selection of journals (inclusive of 9 subjects, more than 200 journals)

Providing 24-hour high-quality service

User-friendly online submission system

Fair and swift peer-review system

Efficient typesetting and proofreading procedure

Display of the result of downloads and visits, as well as the number of cited articles

Maximum dissemination of your research work

Submit your manuscript at: http://papersubmission.scirp.org/

Or contact oja@scirp.org 